\title{
A Comprehensive Survey on Aspect Based Word Embedding Models and Sentiment Analysis Classification Approaches
}

\author{
Monika Agrawal a,1 and Nageswara Rao Moparthi ${ }^{\text {a }}$ \\ a Department of Computer Science \& Engineering, Koneru Lakshmaiah Education \\ Foundation, Greenfields, Vaddeswaram, Guntur- 522502, AP, India
}

\begin{abstract}
Sentiment Analysis includes methods and techniques for businesses to understand and analyze customer reviews, feedback and opinion on a particular product or service. Sentiment Analysis uses Natural Language Processing (NLP) tools to analyze feelings or emotions, attitudes, opinions, thoughts, etc. behind the words. Sentiments such as positive, negative and neutral are associated with a particular product. Sentiment analysis is applicable in multi- domains such as customer feedback for a particular product, movie reviews, social and political comments. This survey basically focuses on different aspect-based word embedding models and aspect-based sentiment classification techniques, where the goal is to extract key features from the sentences and classify sentiment on entities at document level. Aspect Based Sentiment Analysis (ABSA) is a technique that concentrates not only the entire sentence but analyses key terms explicitly to predict the polarity as a whole. ABSA model accepts aspect categories and its corresponding aspect terms to generate sentiment corresponding to each aspect from the text corpus. This article provides a comprehensive survey on different word embedding models under CNN framework for aspect extraction and different machine learning techniques applicable for sentiment classification purpose.
\end{abstract}

Keywords- Aspect sentiment, filtering, classification, polarity.

\section{Introduction}

As the use of internet is growing rapidly, number of users is exchanging their thoughts and opinions on numerous issues on ecommerce and other websites such as twitter, LinkedIn, Amazon, eBay, etc. This social Websites allows users to give their feedback or opinion on use of different products and services, stock exchange data etc., which was very hard to collect desired data and analyze them in an easy way in past time, is now readily available. Through these sites people are sharing their views, feelings and opinion anytime on a particular product or service expressed in positive, negative, or neutral way. Sentiment Classification, is a technique to detect the polarity of given text, is one of the basic tasks in NLP. Figure 1, illustrates General Sentiment Classification process.

\footnotetext{
${ }^{1}$ Monika Agrawal, Department of Computer Science \& Engineering, Koneru Lakshmaiah Education Foundation, India

Email: monika.agrawal1986@gmail.com.
} 


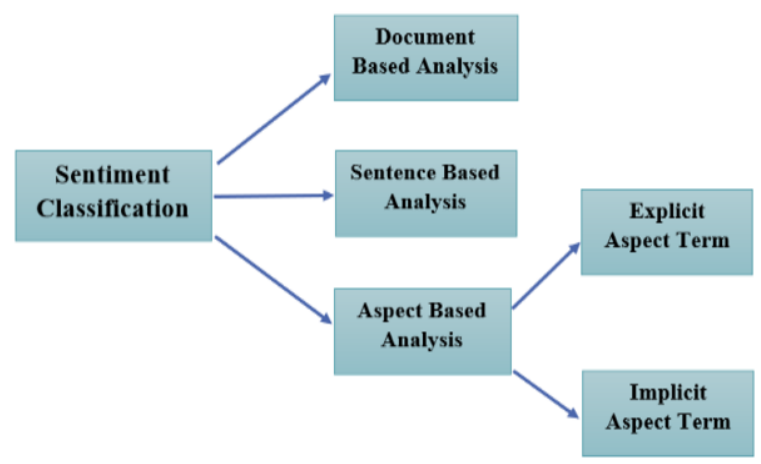

Figure 1. General Sentiment Classification Process

In Document based analysis, Sentiment classification technique extracts all opinion words from the entire document to give the opinion/ sentiment of entire document as a whole. In Sentence based perspective, Sentiment Analysis focusses mainly on polarity detection of complete sentence as positive opinion or negative opinion without considering in to account the important key features of the sentences. Next, Aspect Based Sentiment analysis aims to extract essential aspect related key features from the text and then assigns polarity or classifies sentiment of aspect terms to be positive negative or neutral. In ABSA, first aspect extraction process takes place in which key features are extracted for the given entity and then key features are subjected to machine learning classification approaches to determine the polarity of text [1].

\section{Methodology}

Aspect based sentiment analysis (ABSA) works on three different phases. Figure 2 shows the flowchart of ABSA framework. Consider input data set such as Twitter Dataset, Amazon Product Data, Movies Review Dataset, Paper Reviews dataset etc. First phase begins with Pre-processing or filtering input data using standard NLP library. Filtered data of the phase one is given to the second phase for essential features extraction. In the second phase, word embedding models are designed and implemented on the filtered data for aspect features identification [2].

In this framework, different hybrid word embedding methods such as TF-ID word2vec, GLOVE and skip gram are used to perform feature selection in the deep learning CNN/ RNN framework [3]. The features that are marked in the second phase are given to third phase for text classification process. In the last phase, ML classification techniques are used on high dimensional feature space to detect polarity of given input sentences [4].

\section{Sub Task-2: Aspect Based Feature Extraction}

\subsection{Word2vec}

Word2Vec is a word embedding deep learning model which accepts the preprocessed sentences into tokens and identifies the context of words [5]. This model works on large set of databases including number of documents from different domains like twitter, Facebook, ecommerce websites and returns a huge vector space of several 
dimensions. In this model, each word in the text corpus will be defined by a unique vector in the vector space [6].

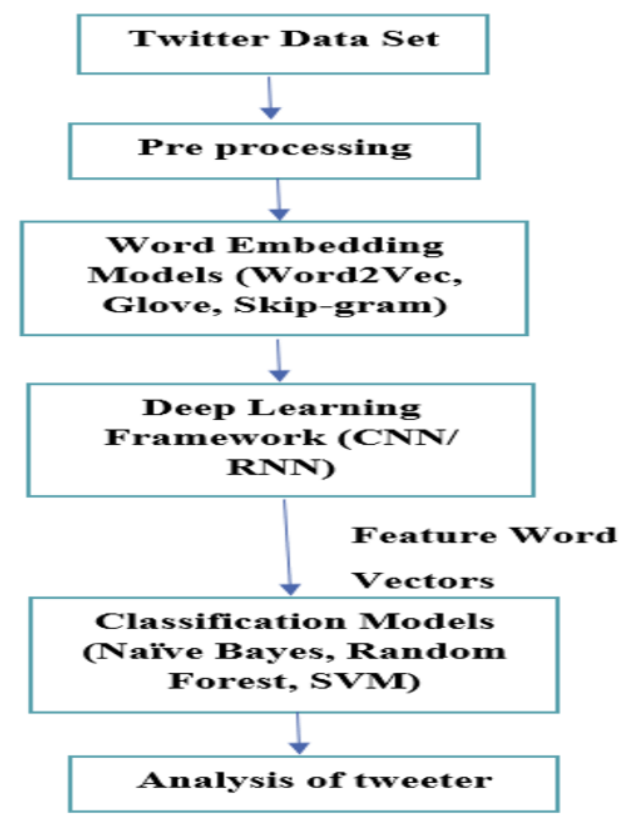

Figure 2. Aspect Based Sentiment Analysis Framework

\subsection{Glove}

Glove generates word embedding by training a model based on global co-occurrence counts of words, global statistics and uses mean squared error as the loss function. The generated word embedding with such a model preserves word relationships and similarities. A co-occurrence matrix in table 2 for a given sentence tells us how often a given pair of words appear together. Each element in the matrix is the count of the pair of the words occurring together [7].

\subsection{Convolution Neural Networks (CNN)}

Convolutional Neural Networks (ConvNets) have in the past years shown breakthrough results in some NLP tasks, one particular task is sentence classification, i.e., classifying short phrases (i.e., around 20 50 tokens), into a set of pre-defined categories. A Convolutional Neural Network typically involves two operations, which can be thought of as feature extractors: convolution and pooling [8].

\section{Sub Task-3: Aspect Based Sentiment Classification}

Most vital activity in sentiment analysis is to identify best suitable classification technique in order to detect polarity of the input text [9]. This section defines, compares and summarizes most of the sentiment classification techniques. Machine learning classification models are one of the best techniques for analyzing huge amount of text data to classify sentiments either as positive, negative or neutral. ML 
Classification algorithms can be categorized as Supervised and Unsupervised learning models. These models gain popularity due to its adaptability and accuracy feature [10].

\subsection{Naïve Bayes}

Naive Bayes is a simple probabilistic technique in which each word of given input text data has probability associated of being positive or negative polarity. In mathematical terms, $\mathrm{P}(\mathrm{A} / \mathrm{B})$ probability of $\mathrm{A}$, if $\mathrm{B}$ is true, is defined as $\mathrm{P}(\mathrm{B} / \mathrm{A})$ times the probability of $A$ being true $\mathrm{P}(\mathrm{A})$, divided by the probability of $\mathrm{B}$ being true $\mathrm{P}(\mathrm{B})$

\subsection{Support Vector Machines}

SVM is a supervised machine learning algorithm can be used for two group classification problems, regression and outlier detection [11]. SVM works on input trained datasets by plotting the points in 2D plane for each category and then draw a hyperplane that best separates the class labels.

\subsection{Neural Networks}

Artificial Neural networks are inspired by human brain neurons, which works on multiple layers and at each layer a number of processing units are defined. The output of first layer is fed as an input to the next consecutive layer and so on. Neural network can learn to perform tasks (e.g., classification) by modifying the connection weights between neurons, resembling the learning process of a biological brain [12].

\subsection{Random Forests}

Random Forest is a Supervised learning technique which works on ensemble learning approach. In this approach, we can either construct a hybridization of similar algorithms number of times or different algorithms can be ensembled. This will result in a dense forest by adding multiple times decision tree algorithms. Random forests are well suited for regression or classification analysis [13].

\section{Evaluation and Comparison of Results}

Table 1, describes the efficiency of the aspect sentiment classification of the different classification models on the input training data. Random forest achieves better efficiency than the other conventional models on training dataset.

Table 1. Comparative analysis of different classification models and its accuracy on aspect sentiment classification

\begin{tabular}{|l|l|l|c|c|}
\hline Test Data & NN & Naïve Bayes & SVM & $\begin{array}{l}\text { Random } \\
\text { Forest }\end{array}$ \\
\hline Aspect Test 1 & 0.82 & 0.86 & 0.82 & 0.92 \\
\hline Aspect Test 2 & 0.8 & 0.86 & 0.84 & 0.91 \\
\hline Aspect Test 3 & 0.83 & 0.84 & 0.86 & 0.91 \\
\hline Aspect Test 4 & 0.81 & 0.85 & 0.86 & 0.91 \\
\hline Aspect Test 5 & 0.84 & 0.86 & 0.85 & 0.93 \\
\hline Aspect Test 6 & 0.84 & 0.86 & 0.87 & 0.92 \\
\hline Aspect Test 7 & 0.84 & 0.86 & 0.85 & 0.92 \\
\hline Aspect Test 8 & 0.81 & 0.87 & 0.88 & 0.91 \\
\hline Aspect Test 9 & 0.82 & 0.84 & 0.86 & 0.92 \\
\hline Aspect Test 10 & 0.81 & 0.86 & 0.85 & 0.92 \\
\hline
\end{tabular}


Table 2, describes the efficiency of the aspect sentiment classification accuracy of the different classification models on the input training data. Random forest achieves better accuracy than the other conventional models on training dataset.

Table 2. Comparative analysis of different classification models and its recall on aspect sentiment classification

\begin{tabular}{|l|c|c|c|c|}
\hline Test Data & NN & $\begin{array}{l}\text { Naïve } \\
\text { Bayes }\end{array}$ & SVM & $\begin{array}{l}\text { Random } \\
\text { Forest }\end{array}$ \\
\hline Aspect Test 1 & 0.83 & 0.86 & 0.87 & 0.92 \\
\hline Aspect Test 2 & 0.82 & 0.86 & 0.83 & 0.92 \\
\hline Aspect Test 3 & 0.81 & 0.87 & 0.88 & 0.92 \\
\hline Aspect Test 4 & 0.82 & 0.84 & 0.85 & 0.92 \\
\hline Aspect Test 5 & 0.81 & 0.84 & 0.83 & 0.91 \\
\hline Aspect Test 6 & 0.82 & 0.84 & 0.86 & 0.91 \\
\hline Aspect Test 7 & 0.83 & 0.85 & 0.83 & 0.91 \\
\hline Aspect Test 8 & 0.81 & 0.85 & 0.85 & 0.92 \\
\hline Aspect Test 9 & 0.84 & 0.84 & 0.83 & 0.91 \\
\hline Aspect Test 10 & 0.8 & 0.84 & 0.82 & 0.91 \\
\hline
\end{tabular}

Figure 3, describes the efficiency of the aspect sentiment classification recall of the different classification models on the input training data. Random forest achieves better recall rate than the other conventional models on training dataset.

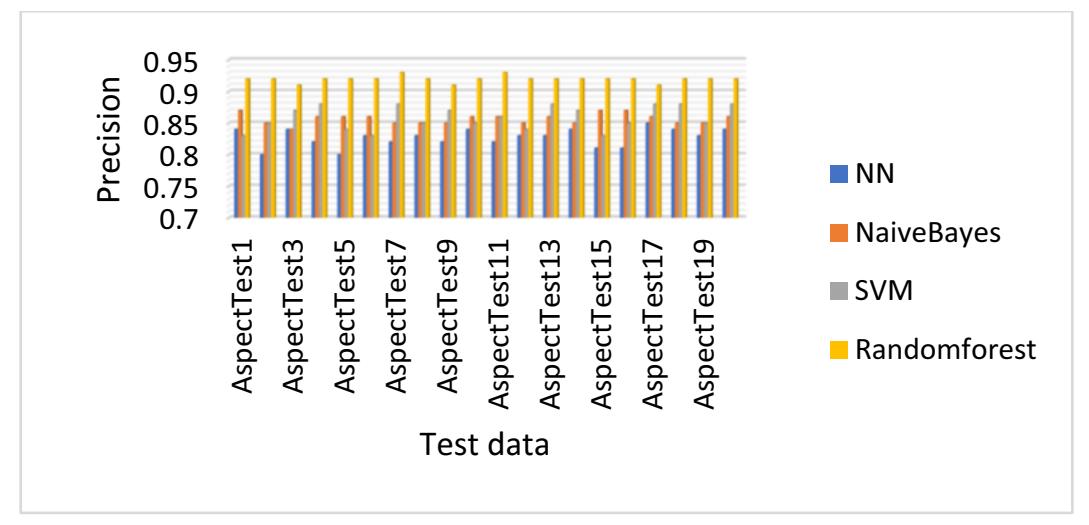

Figure 3. Comparative analysis of different classification models and its precision on aspect sentiment classification

Figure 4, describes the efficiency of the aspect sentiment classification F-measure of the different classification models on the input training data. Random forest achieves better F-measure rate than the other conventional models on training dataset. 


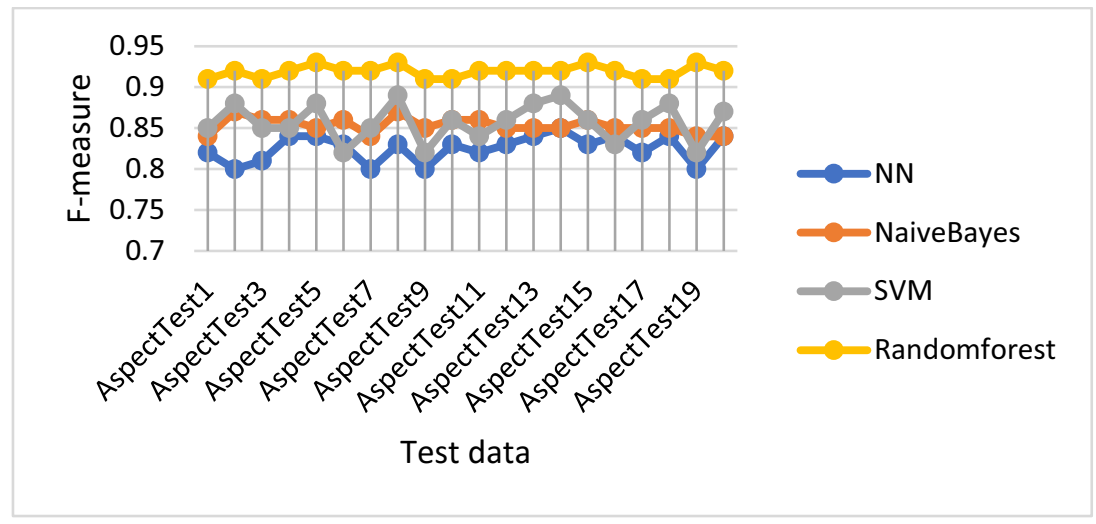

Figure 4. Comparative analysis of different classification models and its F-measure on aspect sentiment classification

\section{Evaluation and Comparison of Results}

In this paper, different aspect sentiment classification models are studied on the input training data for statistical analysis. Most of the conventional classification models are difficult to find the classification efficiency due to problem of feature extraction or noise elimination problem. In this paper, different classification models are tested by integrating the word embedding measures for data classification problem. Experimental results proved that the random forest achieves better accuracy, recall and error rate than

\section{References}

[1] María del Pilar Salas-Zárate, José Medina-Moreira etal, "Sentiment Analysis on Tweets about Diabetes: An Aspect-Level Approach", Hindawi Computational and Mathematical Methods in Medicine Volume 2017, Article ID 5140631, 9 pages https://doi.org/10.1155/2017/5140631

[2] Naveen Kumar Laskari, Suresh Kumar Sanampudi, “Aspect Based Sentiment Analysis Survey”, IOSR Journal of Computer Engineering (IOSR-JCE) e-ISSN: 2278-0661, p-ISSN: 2278-8727, Volume 18, Issue 2, Ver. I (Mar-Apr. 2016), PP 24-28

[3] Nipuna Upeka Pannala, Chamira Priyamanthi Nawarathna, J.T.K.Jayakody, "Supervised Learning Based Approach to Aspect Based Sentiment Analysis", 2016 IEEE International Conference on Computer and Information Technology.

[4] M.Sivakumar, Dr.U.Srinivasulu Reddy, “Aspect Based Sentiment Analysis of Students Opinion using Machine Learning Techniques”, Proceedings of the International Conference on Inventive Computing and Informatics (ICICI 2017) IEEE Xplore.

[5] Alson Cahyadi and Masayu Leylia Khodra, "Aspect-Based Sentiment Analysis Using Convolutional Neural Network and Bidirectional Long Short-Term Memory”, 978-1-5386-4804-9/18/\$31.00@2018 IEEE

[6] Ganpat Singh Chauhan, Yogesh Kumar Meena, "An Unsupervised Multiple Word-Embedding Method with Attention Model for Cross Domain Aspect Term Extraction", 3rd International Conference on Emerging Technologies in Computer Engineering: Machine Learning and Internet of Things (ICETCE2020), 07-08 February 2020

[7] Walaa Medhat, Ahmed Hassan, Hoda Korashy, "Sentiment analysis algorithms and applications: A survey", Ain Shams Engineering Journal, 2014

[8] Lei Zhang, Shuai Wang, Bing Liu, "Deep Learning for Sentiment Analysis: A Survey”, Wiley, January 2018, DOI: 10.1002/widm.1253

[9] Toqir A. Rana, Yu-N Cheah, "Aspect extraction in sentiment analysis: comparative analysis and survey", Published in Springer 2016, DOI 10.1007/s10462-016-9472-z. 
[10] A.M.Abirami, V.Gayathri, "A Survey On Sentiment Analysis Methods And Approach" 2016 IEEE Eighth International Conference on Advanced Computing (ICoAC)

[11] Oscar Araque, Ganggao Zhu, Manuel Garcia-Amado and Carlos A. Iglesias, "Mining the Opinionated Web: Classification and Detection of Aspect Contexts for Aspect Based Sentiment Analysis", 2016 IEEE 16th International Conference on Data Mining Workshops.

[12] Ruijun Liu, Yuqian Shi, Changjiang Ji, And Ming Jia, "A Survey of Sentiment Analysis Based on Transfer Learning”, IEEE Access, 2019 DOI: 10.1109/ACCESS.2019.2925059

[13] Soujanya Poria, Erik Cambria, Alexander Gelbukh, "Aspect Extraction for Opinion Miningwith a Deep Convolutional Neural Network" Knowledge-Based Systems Published by Elsevier, 2016 DOI: 10.1016/j.knosys.2016.06.009 